\title{
Immunoglobulin G4-related epidural inflammatory pseudotumor presenting with pulmonary complications and spinal cord compression: case report
}

\author{
Kavelin Rumalla, ${ }^{1}$ Kyle A. Smith, MD, ${ }^{2}$ and Paul M. Arnold, MD² \\ ${ }^{1}$ School of Medicine, University of Missouri-Kansas City, Missouri; and 'Department of Neurosurgery, University of Kansas \\ Medical Center, Kansas City, Kansas
}

Immunoglobulin G4-related disease (IgG4-RD) is a recently defined condition characterized by inflammatory tumefactive lesions in various organ systems. IgG4-RD is a clinical and radiological diagnosis of exclusion and requires the presence of specific histopathological criteria for diagnosis.

A 50-year-old man presented to an outside hospital with a 3-month history of progressively worsening back pain and symptoms of pleurisy, nasal crusting, and hematochezia. Radiological workup revealed an epidural-paraspinal mass with displacement of the spinal cord, destruction of the T5-6 vertebrae, and extension into the right lung. Biopsy sampling and subsequent histopathological analysis revealed dense lymphoplasmacytic infiltrate with an increased number of IgG4-positive plasma cells and a storiform pattern of fibrosis. With strong histopathological evidence of lgG4-RD, the patient was started on a regimen of prednisone. Further testing ruled out malignant neoplasm, infectious etiologies, and other autoimmune diseases. Two weeks later, the patient presented with acute-onset paraplegia due to spinal cord compression. The patient underwent decompression laminectomy of T5-6, posterior instrumented fusion of T2-8, and debulking of the epidural-paraspinal mass. After the continued administration of glucocorticosteroids, the patient improved remarkably to near-normal strength in the lower extremities and sensory function 6 months after surgery.

To the authors' knowledge, this is the first case of lgG4-related epidural inflammatory pseudotumor and spinal cord compression in the United States. This case highlights the importance of early administration of glucocorticosteroids, which were essential to preventing further progression and preventing relapse. IgG4-RD evaluation is important after other diseases in the differential diagnosis are ruled out.

https://thejns.org/doi/abs/10.3171/2016.11.SPINE16924

KEY WORDS IgG4; inflammatory pseudotumor; lymphoplasmacytic infiltrate; epidural mass; thoracic spine

I MMUNOGLOBUlin G4-related disease (IgG4-RD) is a newly classified and increasingly recognized fibroinflammatory condition characterized clinically by tumefactive lesions in a wide range of organ systems. ${ }^{4,11,20}$ IgG4 disease has been described in nearly every organ system, with varying pathological features. ${ }^{20}$ The most common sites of IgG4-RD involvement are the pancreas (35\%), bile duct (25\%), and lungs (15\%). ${ }^{18}$ The differential diagnosis of IgG4-RD requires exclusion of other diagnoses via clinical and radiological evidence followed by biopsy and confirmation of IgG4-RD through histopathological analysis. An elevated level of IgG4 antibody in the serum is not a strong diagnostic factor in terms of sensitivity and specificity and may or may not be pres- ent in cases of IgG4-RD.,20 The condition is diagnosed histopathologically by the presence of dense lymphoplasmacytic infiltrate, storiform-patterned fibrosis, and obliterative phlebitis. ${ }^{4,20}$ Since the criteria for IgG4-RD were defined, many previously identified autoimmune diseases are now considered part of the IgG4 family of diseases, such as inflammatory pseudotumor (IPT). ${ }^{20}$

Inflammatory pseudotumors (IPTs) are benign lesions of unknown etiology that mimic the appearance of malignant tumors and are found in various regions of the body. ${ }^{14}$ However, after IgG4-RD was defined, further investigation revealed that a significant percentage of IPT lesions contained IgG4-positive plasma cell infiltration and were actually manifestations of IgG4-RD. ${ }^{22,23}$ A total of 9 cases

ABBREVIATIONS IgG4-RD = immunoglobulin G4-related disease; IMT = inflammatory myofibroblastic tumor; IPT = inflammatory pseudotumor. SUBMITTED August 4, 2016. ACCEPTED November 11, 2016.

INCLUDE WHEN CITING Published online March 17, 2017; DOI: 10.3171/2016.11.SPINE16924. 
of IPT involving the epidural space have been reported in the literature..$^{5,6,9,10,12,17,19}$ However, only one of these IPT cases had a confirmed diagnosis of IgG4-RD. ${ }^{5}$

We present a rare case of a patient with IgG4-related epidural IPT with extension through the thoracic vertebrae into the right lung. This IgG4-related IPT required resection of the pulmonary mass, emergency decompression of the spinal cord, and administration of high doses of glucocorticosteroids. To our knowledge, this is the first reported case of IgG4-RD of the epidural space to cause destruction of the thoracic vertebrae and the first case of epidural IgG4-RD reported in the United States.

\section{Case Report}

History

A 50-year-old man with no significant medical history presented with a 3-month history of rapidly progressive, severe back pain localized to the T-6 level. Based on normal findings on radiography, a primary care provider managed the patient's pain conservatively. One month later, the patient began experiencing shortness of breath with severe pain. Additional associated symptoms included nasal crusting, bloody stool, and pleuritic chest pain. CT scanning of the chest at an outside hospital revealed a $4-\mathrm{cm}$ mass centered at the superior segment of the right lung lower lobe with destruction of the T-5 and T- 6 vertebral bodies (Fig. 1). Follow-up MRI study of the thoracic spine demonstrated bony involvement of the T4-6 vertebrae with epidural extension into the right lung and avid enhancement, spinal cord displacement without compression, and surrounding soft-tissue involvement anterolaterally to the right; in addition, mild loss of height at T-6 was seen because of a pathologic compression fracture (Fig. 2). The patient's care was managed at an outside hospital with further workup, including a needle biopsy of the lung mass. Biopsy results demonstrated lymphoplasmacytic infiltrate with a significant increase in IgG4-positive plasma cells within fibrosis and foci of atypical cells, negative for infection (Fig. 3). Informed consent was obtained prior to the biopsy procedure. Prednisone $40 \mathrm{mg}$ daily was initiated 1 week prior to presentation to our institution's rheumatology service. During the week that followed, the patient's back pain continued to worsen, and no improvement in other symptoms, including shortness of breath, was noted despite prednisone. Further workup was completed for autoimmune-inflammatory disease, and prednisone was increased to $60 \mathrm{mg}$ daily.

Two weeks later, the patient presented to our emergency department with acute onset of paraplegia and urinary retention. His weakness progressed over the course of several hours prior to presentation on the same day.

\section{Examination}

Lower-extremity examination demonstrated $0 / 5$ strength bilaterally and a sensory level at T-6. The patient's reflexes were present but not brisk. Rectal tone was intact with positive bulbocavernous reflex. Repeat MRI at this time demonstrated a pathologic fracture involving T5-6 with significant canal stenosis and spinal cord compression (Fig.4).
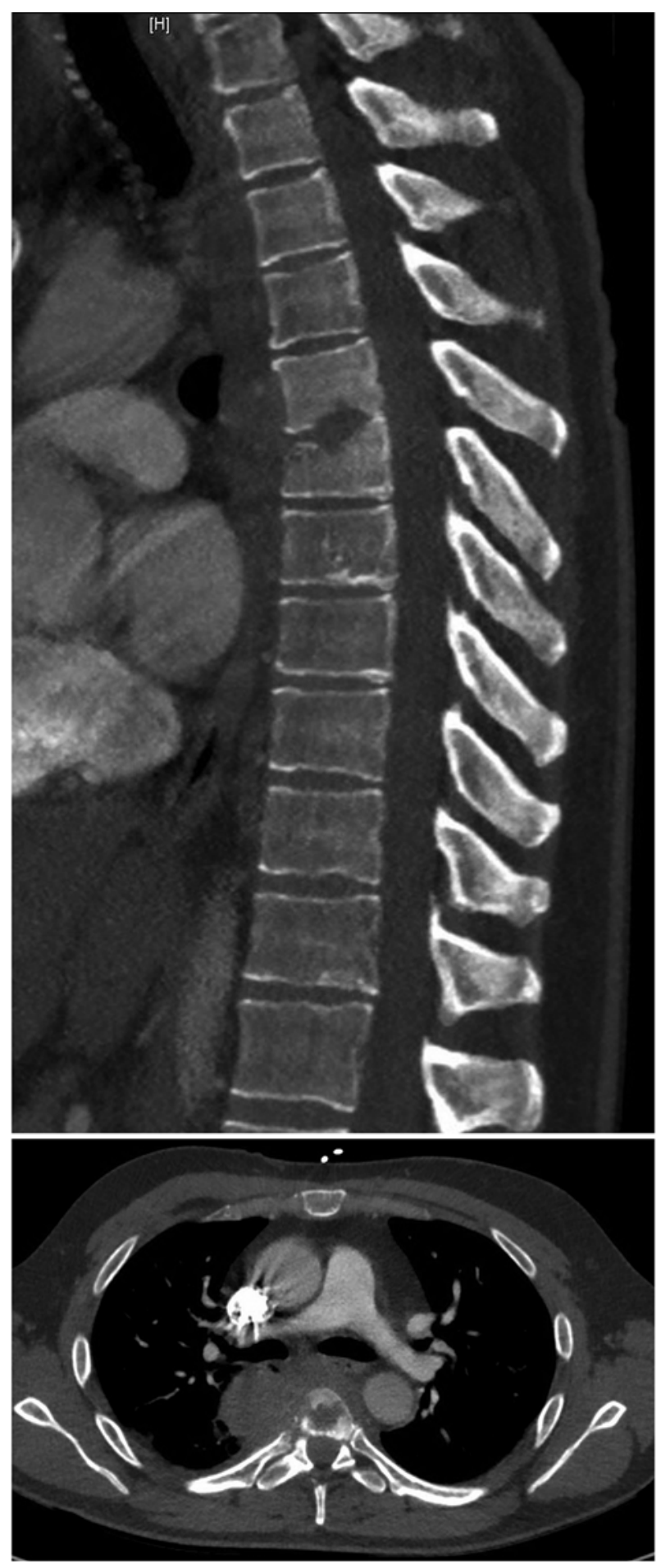

FIG. 1. Preoperative sagittal (upper) and axial (lower) chest CT sequences demonstrating a 4-cm mass centered at the superior segment of the right lung lower lobe with bony destruction of the T5-6 vertebral bodies.

\section{Operation}

After admission on the same day, the patient underwent a thoracic T5-6 laminectomy with T2 -8 posterior instrumented fusion and debulking of the epidural-paraspinal mass (Fig. 5). Informed consent for treatment was 


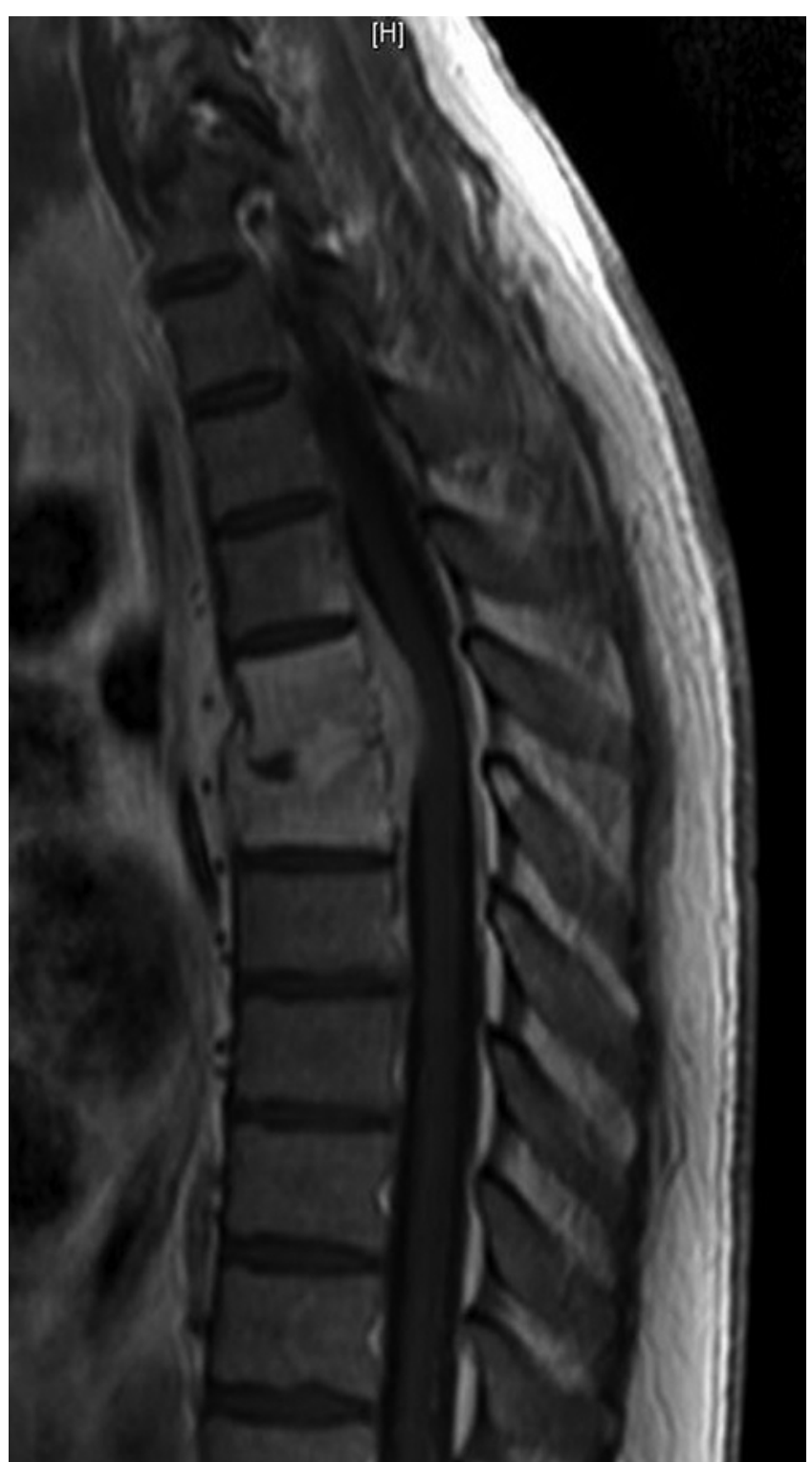

FIG. 2. Preoperative sagittal MR image of the thoracic spine demonstrating a contrast-enhancing mass at T5-6 with bony involvement and epidural extension.

obtained prior to intervention. Intraoperative specimens were sent for both frozen and permanent pathology. $\mathrm{Pu}-$ rulence was noted at the T-6 level, and intraoperative cultures were sent.

\section{Postoperative Course}

Intraoperative culture demonstrated methicillin-sensitive Staphylococcus aureus cultured from the wound at the site of prior mass resection. Examination of the patient's laboratory specimens revealed normal levels of serum IgG4 concentration. Long-term nafcillin was initiated. Approximately 2 weeks later, the patient developed decreased strength and was found to have a large subcutaneous paraspinal fluid collection. This collection was percutaneously drained $(610 \mathrm{ml})$ and proved to be sterile by culture. Beta- 2 transferrin was negative, confirming

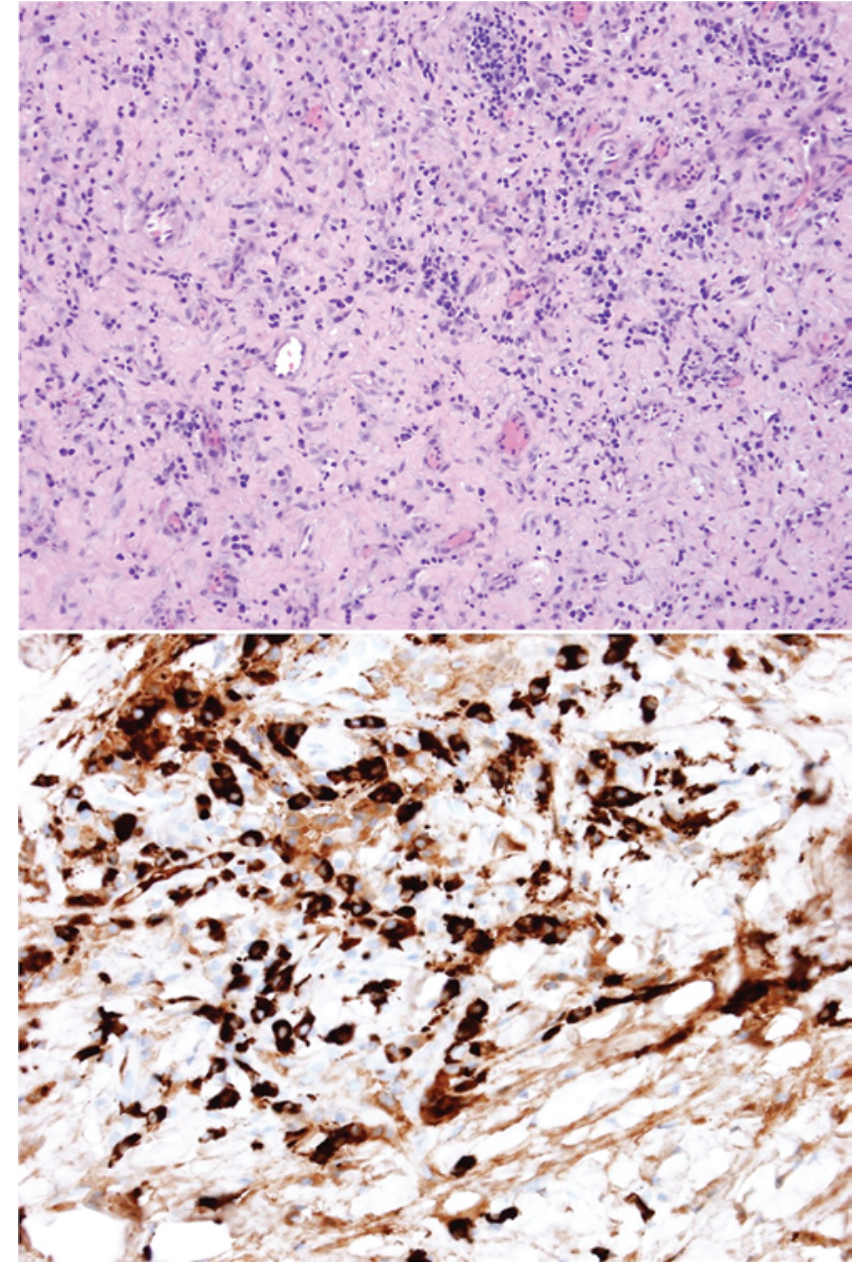

FIG. 3. Photomicrographs of pathology specimens demonstrating fibroinflammatory consolidation. Upper: A significant number of lgG4-positive plasma cells and foci of atypical cells are seen. $\mathrm{H}$ \& $\mathrm{E}$. Lower: IgG4 immunohistochemistry stain demonstrating a significant number of IgG4-positive cells. Original magnification $\times 400$ (upper); $\times 600$ (lower). Figure is available in color online only.

the likely diagnosis of seroma. The fluid again accumulated within several days, and the patient was admitted for percutaneous drain placement adjacent to the right pleural space with $430 \mathrm{ml}$ initial drainage. During hospitalization, the patient's lower-extremity strength improved to $3 / 5$ proximally to $5 / 5$ distally. On follow-up approximately 2 months later, the patient's lower-extremity strength was near normal. Intravenous antibiotics were continued for a 12-week course with oral suppressive antibiotics for 1 year, given the presence of spinal hardware. Steroid immunosuppression was continued but tapered to a low dose for IgG4 disease until imaging follow-up to assess response.

\section{Discussion}

IgG4-RD is a newly emerging disease that is a collection of syndromes previously thought to be unrelated but are now known to share common clinical, radiological, and histopathological features. The disease has been clinically and radiologically characterized as diffuse or local- 


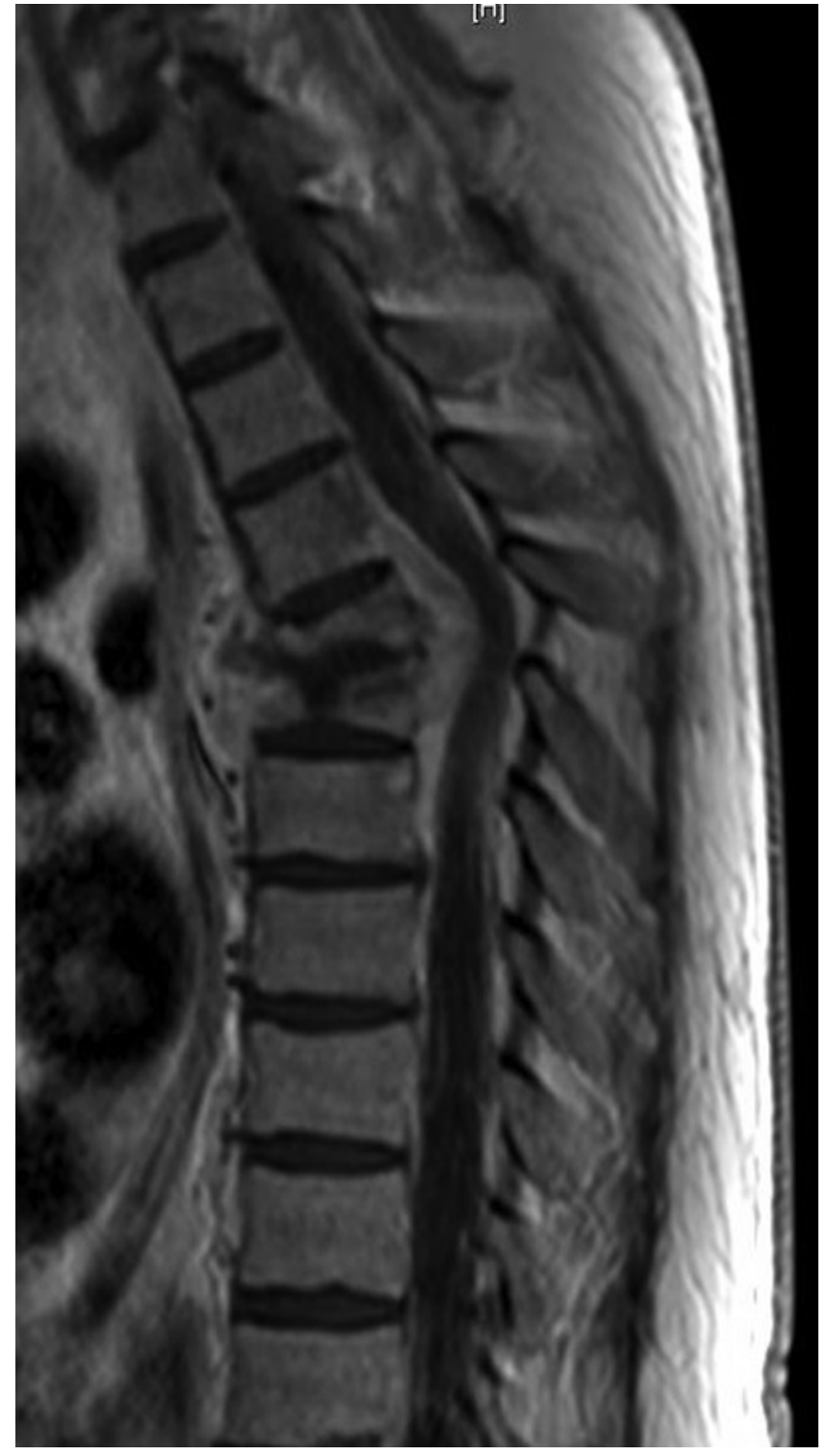

FIG. 4. Preoperative sagittal MR image of the thoracic spine demonstrating progression of T5-6 bony destruction with significant canal compromise and kyphosis.

ized masses in single or multiple organs..$^{18,21}$ It presents with similar signs and symptoms of malignant neoplasm, infectious disease, and autoimmune disease, making it a clinical and radiological disease of exclusion and a histopathological disease of inclusion. ${ }^{4,20}$

Clinical findings, radiological evidence, and serum concentration of IgG4 are not sensitive or specific for the diagnosis of IgG4-related IPT, making diagnosis particularly difficult. ${ }^{4,20}$ Furthermore, many other diagnoses must be eliminated from the differential diagnosis before IgG4related IPT is suspected. Since our patient presented with suspected IgG4-related IPT of the epidural space with extension into the lungs, we had to consider 2 separate differential diagnoses. The differential for IgG4-related disease of the lungs includes malignancy, inflammatory myofibroblastic tumor, sarcoidosis, Castleman's disease,

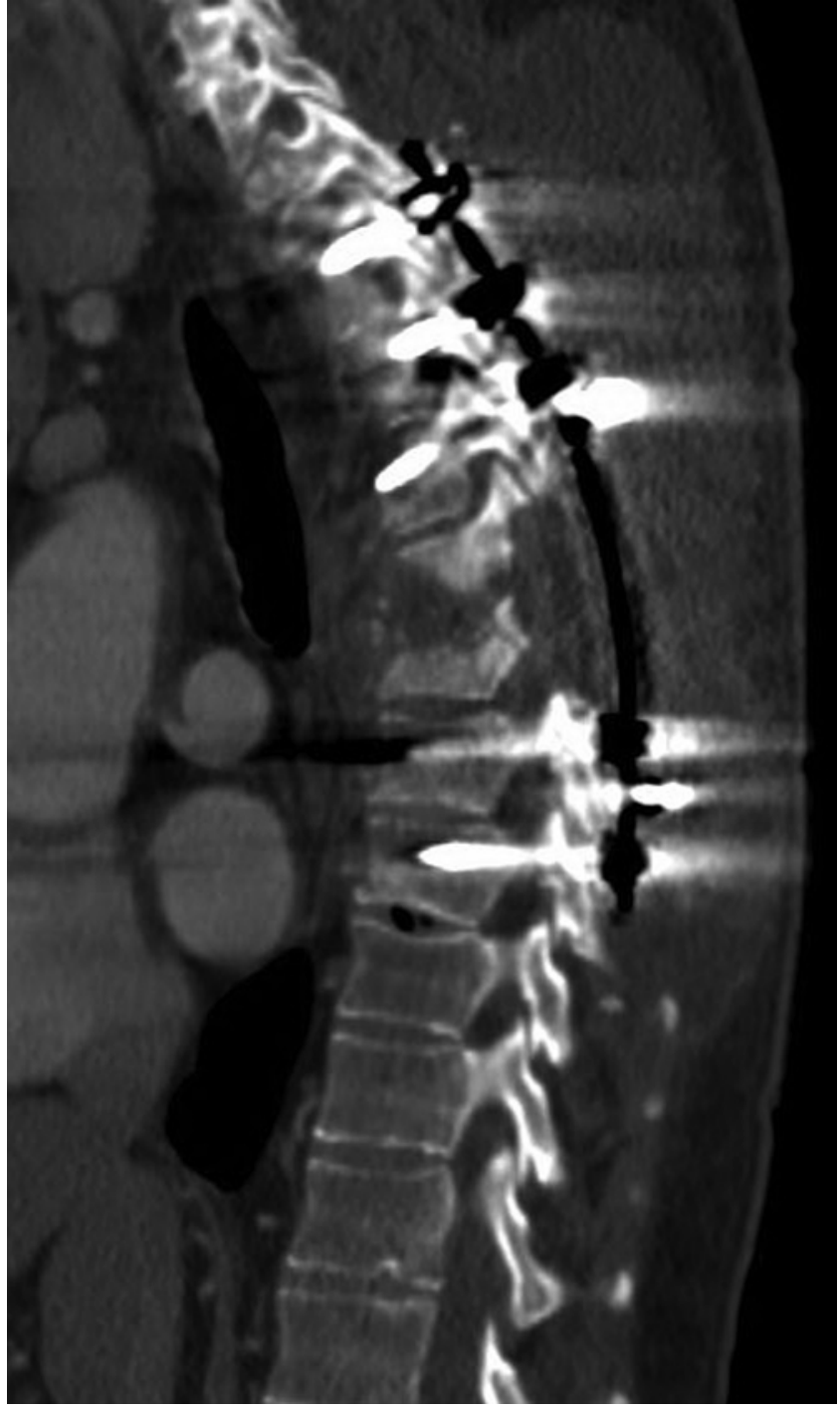

FIG. 5. Postoperative sagittal CT scan of the thoracic spine, demonstrating postoperative changes and posterior instrumented fusion.

lymphomatoid, granulomatosis, idiopathic interstitial pneumonia, and Erdheim-Chester disease. ${ }^{20}$

The possibility of other autoimmune diseases and infectious causes were excluded after examination of the patient's laboratory results and pathology findings. It is impossible to distinguish between malignant neoplasms and IPTs based on clinical and radiological evidence. ${ }^{4,20}$ This distinction requires biopsy and subsequent histopathological analysis. The histopathology report for our patient did not reveal the presence of lymphoma or malignant neoplasm. Lymphomas appear very similar to IgG4-related IPT in histopathological analysis and must be ruled out using clonality studies. ${ }^{20}$ In lymphoma, the lymphoplasmacytic infiltrate would contain predominantly B-cells, whereas the presence of IgG4-RD is marked by predominance of T-cells and diffuse infiltrates of IgG4-positive plasma cells. ${ }^{20}$

The 2 main types of IPT are pulmonary inflammatory myofibroblastic tumor (IMT) and IgG4-related pulmonary 
inflammatory pseudotumor (IPT). IPT and IMT share nearly identical clinical and radiological features and can only be distinguished histopathologically. This distinction is important because IMT is typically treated surgically, whereas IPT is treated conservatively. ${ }^{2}$ Distinct characteristics of IPT compared with IMT include the lack of anaplastic lymphoma kinase expression, obstructive phlebitis, more prominent lymphoplasmacytic infiltrate than spindle cell proliferation, and increased levels of IgG4 expression in plasma cells. Based on these criteria, we can exclude the possibility that our patient had IMT.

A consensus on the histopathological diagnosis of IgG4-RD was reached among a committee of 35 experts from several countries at the international symposium on IgG4-RD. ${ }^{4,20}$ According to this consensus, the diagnosis of IgG4-RD is dependent on the presence of increased numbers of IgG4-positive plasma cells in the tissue infiltrate in addition to specific histopathological features. The key histopathological features include 1) dense lymphoplasmacytic infiltrate, 2) storiform pattern of fibrosis, and 3) obliterative phlebitis.,20 A confident pathological diagnosis of IgG4-RD only requires the presence of 2 of these 3 criteria, most often encompassing the first two. ${ }^{4}$ Our case involved dense lymphoplasmacytic infiltrate, a storiform pattern of fibrosis, and increased levels of IgG4positive plasma cells in the tissue, suggesting a diagnosis of IgG4-related epidural IPT with secondary expansion into the lung. The patient's serum IgG4 levels measured after 2 months of glucocorticosteroid administration were normal, but they may have been initially elevated at the time the agents were first administered. The serum IgG4 level is not known as a sensitive diagnostic marker and is normal in $25 \%-30 \%$ of patients with confirmed IgG4RD. ${ }^{4,8,18,20}$ Furthermore, elevations in IgG4 serum concentration are not specific for IgG4-RD and can be elevated in other disorders such as sarcoidosis and allergies. ${ }^{1}$

\section{Treatment}

One of the major predictors of outcomes after treatment for IgG4-RD is the degree of fibrosis at the time of treatment. ${ }^{20}$ Thus, once lymphoplasmacytic inflammation is present, aggressive treatment is important to prevent extensive fibrosis and subsequent severe organ dysfunction. ${ }^{20}$ Glucocorticosteroid treatment is known to be the first-line treatment for suspected and confirmed IgG4-RD, and relapse rates are known to be lower when maintenance therapy is present. ${ }^{18,20}$ In the analysis by Sekiguchi et al., more than $40 \%$ of patients treated with corticosteroid therapy experienced relapse of disease. ${ }^{18}$ Our patient was treated with glucocorticosteroids (prednisone) as soon as IgG4RD was suspected. Glucocorticsteroids are the first-line treatment for this disease; in retrospect, one may question whether surgery could have prevented neurological decline. However, in an intact patient, current literature guides medical treatment. Other modalities including bracing for the compression fracture could have been considered, but the patient's neurological decline occurred prior to presentation at our institution. Tissue cultures obtained during the operation that were sent for pathological examination were positive for methicillin-resistant $S$. aureus, suggesting that the patient's deteriorating lower- extremity motor and sensory function could have been related to $S$. aureus osteomyelitis adjacent to the site of pulmonary mass resection. Thus, it is unclear how much IgG4-RD in comparison with possible osteomyelitis contributed to kyphosis and collapse and the resultant decline that was seen during neurological examination. The initial biopsy/subtotal resection was likely within the pleural cavity and did not involve the vertebral bodies themselves.

Based on the patient's improved lower-extremity strength at the 2-month follow-up, the steroid immunosuppression appeared to successfully prevent further progression and/or prevent relapse of the IgG4-related epidural IPT. In cases of recurrent or refractory disease, rituximab has been shown to be an effective treatment for IgG4$\mathrm{RD}$, even without concomitant glucocorticosteroid treatment. ${ }^{3,20}$ Azathioprine, ${ }^{15}$ mycophenolate mofetile, ${ }^{13}$ and methotrexate ${ }^{7}$ can also be used as remission-maintenance drugs if glucocorticosteroid treatment is not successful.

\section{Literature Review}

IPT of the epidural space is very rare and, to our knowledge, there have only been 9 previously reported cases

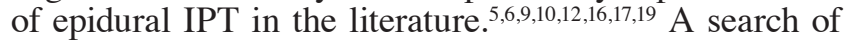
PubMed was completed for case reports and case series involving this disease. Furthermore, only one of these cases had a suspected diagnosis of IgG4-RD. ${ }^{5}$ Ferreira et al. described the case of a patient who presented with severe paraparesis and sensory loss and was admitted to a hospital in Lisbon, Portugal, with biopsy-confirmed IgG4related IPT from T-10 to T-12.5 This patient also required decompression laminectomy and complete resection of the mass. However, the patient experienced recurrence of the epidural mass on 2 separate occasions and required 2 subsequent surgeries. Further analysis of the biopsy specimen revealed lymphoplasmacytic infiltration within fibrosis and increased concentration of IgG4-positive plasma cells.

\section{Conclusions}

IgG4-RD is a newly emerging and increasingly recognized fibroinflammatory condition that encompasses a wide range of previously defined organ-specific conditions. The diagnosis of IgG4-RD requires exclusion of other diseases in the differential diagnosis and the presence of specific histopathological features. To our knowledge, this is the first reported case of IgG4-related epidural IPT in the United States. The IPT in our patient's case was aggressive and resulted in destruction of the T5-6 vertebral bodies, epidural extension and enhancement, spinal cord displacement without compression, and surrounding soft-tissue involvement. The patient underwent decompression laminectomy and posterior instrumented fusion with debulking of the tumor mass to stabilize the thoracic spine and prevent further compression of the spinal cord. Administration of glucocorticosteroids was not effective enough to prevent neurological deterioration in the acute phase, but it may have significant value in preventing growth of smaller residual masses and disease relapse. From the time of first admission to follow-up at 2 months, our patient's symptoms improved remarkably, with lower- 
extremity strength improving from $0 / 5$ to $5 / 5$. Our case suggests that once other conditions, such as malignancy, infection, and other autoimmune diseases, are ruled out, IgG4-RD should be included in the differential diagnosis for a spinal tumor, and glucocorticosteroids should be administered as soon as possible.

\section{Acknowledgments}

We thank Kevin Leslie, MD, of the Mayo Clinic for assistance with obtaining pathology micrographs for this case report.

\section{References}

1. Aalberse RC, Stapel SO, Schuurman J, Rispens T: Immunoglobulin G4: an odd antibody. Clin Exp Allergy 39:469-477, 2009

2. Bhagat P, Bal A, Das A, Singh N, Singh H: Pulmonary inflammatory myofibroblastic tumor and IgG4-related inflammatory pseudotumor: a diagnostic dilemma. Virchows Arch 463:743-747, 2013

3. Carruthers MN, Topazian MD, Khosroshahi A, Witzig TE, Wallace ZS, Hart PA, et al: Rituximab for IgG4-related disease: a prospective, open-label trial. Ann Rheum Dis 74:1171-1177, 2015

4. Deshpande V, Zen Y, Chan JK, Yi EE, Sato Y, Yoshino T, et al: Consensus statement on the pathology of IgG4-related disease. Mod Pathol 25:1181-1192, 2012

5. Ferreira NR, Vaz R, Carmona S, Mateus S, Pereira P, Fernandes L, et al: IgG4-related disease presenting with an epidural inflammatory pseudotumor: a case report. J Med Case Reports 10:61, 2016

6. Gilliard C, De Coene B, Lahdou JB, Boutsen Y, Noël H, Godfraind C: Cervical epidural pseudotumor and multifocal fibrosclerosis. Case report and review of the literature. $\mathbf{J}$ Neurosurg 93 (1 Suppl):152-156, 2000

7. Hyun JW, Kim SH, Yoo H, Hong EK, Huh SY, Kim HJ: Steroid-resistant relapsing IgG4-related pachymeningitis treated with methotrexate. JAMA Neurol 71:222-225, 2014

8. Kamisawa T, Zen Y, Pillai S, Stone JH: IgG4-related disease. Lancet 385:1460-1471, 2015

9. Kanagaraju V, Rai D, Alluri RVC, Prasanna C, Sundar VS, Kumar SMA, et al: An inflammatory pseudotumor in the thoracic epidural space presenting with progressive paraplegia: a histopathological diagnosis with clinical and radiological uncertainty. Case report with literature review. Eur Spine J 25 (Suppl 1):75-79, 2016

10. Kato S, Murakami H, Demura S, Yoshioka K, Okamoto Y, Hayashi $\mathrm{H}$, et al: Epidural inflammatory pseudotumor in the thoracic spine in a patient with polymyalgia rheumatica. Spine J 12:e1-e4, 2012

11. Khosroshahi A, Wallace ZS, Crowe JL, Akamizu T, Azumi A, Carruthers MN, et al: International consensus guidance statement on the management and treatment of IgG4-related disease. Arthritis Rheumatol 67:1688-1699, 2015

12. Kim JS, Park SY: Inflammatory pseudotumor in the epidural space of lumbosacral spine on ${ }^{18} \mathrm{~F}-\mathrm{FDG}$ PET/CT. Asia Ocean J Nucl Med Biol 2:138-142, 2014

13. Moss HE, Mejico LJ, de la Roza G, Coyne TM, Galetta SL, Liu GT: IgG4-related inflammatory pseudotumor of the central nervous system responsive to mycophenolate mofetil. J Neurol Sci 318:31-35, 2012

14. Patnana M, Sevrukov AB, Elsayes KM, Viswanathan C,
Lubner M, Menias CO: Inflammatory pseudotumor: the great mimicker. AJR Am J Roentgenol 198:W217-W227, 2012

15. Pozdzik AA, Brochériou I, Demetter P, Matos C, Delhaye $M$, Devière J, et al: Azathioprine as successful maintenance therapy in IgG4-related tubulointerstitial nephritis. Clin Kidney J 5:225-228, 2012

16. Roberts GA, Eldridge PR, Mackenzie JM: Case report: inflammatory pseudotumour of the spine, with literature review. Br J Neurosurg 11:570-572, 1997

17. Sailler LJ, Porte L, Ollier SM, Astudillo LM, Couret BG, Catalaa I, et al: Giant cell arteritis and spinal cord compression: an overlap syndrome? Mayo Clin Proc 81:89-91, 2006

18. Sekiguchi H, Horie R, Kanai M, Suzuki R, Yi ES, Ryu JH: $\mathrm{IgG} 4$-related disease: retrospective analysis of one hundred sixty-six patients. Arthritis Rheumatol 68:2290-2299, 2016

19. Seol HJ, Kim SS, Kim JE, Lee SH, Won JY: Inflammatory pseudotumor in the epidural space of the thoracic spine: a case report and literature review of MR imaging findings. AJNR Am J Neuroradiol 26:2667-2670, 2005

20. Stone JH, Zen Y, Deshpande V: IgG4-related disease. N Engl J Med 366:539-551, 2012

21. Umehara H, Okazaki K, Masaki Y, Kawano M, Yamamoto M, Saeki T, et al: Comprehensive diagnostic criteria for IgG4-related disease (IgG4-RD), 2011. Mod Rheumatol 22:21-30, 2012

22. Yamamoto H, Yamaguchi H, Aishima S, Oda Y, Kohashi K, Oshiro Y, et al: Inflammatory myofibroblastic tumor versus IgG4-related sclerosing disease and inflammatory pseudotumor: a comparative clinicopathologic study. Am J Surg Pathol 33:1330-1340, 2009

23. Zen Y, Kitagawa S, Minato H, Kurumaya H, Katayanagi K, Masuda S, et al: IgG4-positive plasma cells in inflammatory pseudotumor (plasma cell granuloma) of the lung. Hum Pathol 36:710-717, 2005

\section{Disclosures}

Dr. Arnold reports the following. Commercial relationships with Z-Plasty (stock, stock options, or other ownership interest); Medtronic Sofamor Danek (salary and any payment for services not otherwise identified as salary, such as consulting, fees, honoraria, paid authorship, or other payments for services); Stryker Spine (sponsored or reimbursed travel, salary and any payment for services no otherwise identified as salary such as consulting, fees, honoraria, paid authorship, or other payments for services); AOSpine North America (sponsored or reimbursed travel); Invivo (salary and any payment for series not otherwise identified as salary such as consulting, fees, honoraria, paid authorship, or other payments for services). None of these commercial relationships affected nor provided assistance to this paper.

\section{Author Contributions}

Conception and design: Arnold. Acquisition of data: Arnold, Smith. Analysis and interpretation of data: all authors. Drafting the article: Rumalla, Smith. Critically revising the article: all authors. Reviewed submitted version of manuscript: all authors. Approved the final version of the manuscript on behalf of all authors: Arnold.

\section{Correspondence}

Paul M. Arnold, Department of Neurosurgery, University of Kansas Medical Center, 3901 Rainbow Blvd., Delp 5080, Mail Stop 3021, Kansas City, KS 66160. email: parnold@kumc.edu. 